\title{
Safety and effectiveness of a fixed-dose combination of olmesartan, amlodipine, and hydrochlorothiazide in clinical practice
}

This article was published in the following Dove Press journal:

Vascular Health and Risk Management

17 December 2014

Number of times this article has been viewed

\section{Peter Bramlage \\ Eva-Maria Fronk ${ }^{2}$ \\ Wolf-Peter Wolf ${ }^{3}$ \\ Rüdiger Smolnik ${ }^{3}$ \\ Gemma Sutton' \\ Roland E Schmieder ${ }^{4}$ \\ 'Institut für Pharmakologie und präventive Medizin, Mahlow, Germany; ${ }^{2}$ Daiichi Sankyo Europe GmbH, Munich, Germany; ${ }^{3}$ Daiichi Sankyo Deutschland GmbH, Munich, Germany; \\ ${ }^{4}$ Abteilung für Nephrologie und Hypertensiologie, Universitätsklinikum Erlangen, Erlangen, Germany}

Correspondence: Peter Bramlage Institut für Pharmakologie und präventive Medizin, Menzelstrasse 21,

I583 I Mahlow, Germany

Tel +4933793147890

Fax +493379 3।4 7892

Email peter.bramlage@ippmed.de
Background: Clinical trials indicate that the use of fixed-dose combinations (FDCs) is associated with a higher level of treatment adherence and prolonged blood pressure (BP) control. The aim of this study was to document the safety and effectiveness of the FDC olmesartan/amlodipine/ hydrochlorothiazide in patients with essential hypertension in clinical practice.

Methods: This multicenter, prospective, 24-week, noninterventional study enrolled 5,831 patients from primary care offices in Germany and Austria. Inclusion criteria were a diagnosis of essential hypertension and newly initiated treatment with the FDC.

Results: The mean age of patients was 63.5 years, almost $50 \%$ of patients had a time since diagnosis of essential hypertension of over 5 years, and approximately $70 \%$ of patients had at least one cardiovascular risk factor, including $29.4 \%$ of patients with diabetes mellitus. Following approximately 24 weeks of treatment, the mean reduction in systolic/diastolic BP was $29.0 / 14.0 \mathrm{mmHg}$, a BP response was observed by $94.2 \%$ of patients, and a target $\mathrm{BP}$ of $<140 / 90 \mathrm{mmHg}$ was attained in $67.5 \%$ of patients. At least one adverse drug reaction (ADR) was experienced by $1.2 \%$ of patients, with the most common being peripheral edema. Subanalyses demonstrated that the following factors did not have a significant influence on the ADR rate: age ( $<65$ years versus $\geq 65$ years), diabetes mellitus (no/yes), cardiovascular risk (low/high), and concomitant medication (no/yes).

Conclusion: This study demonstrates that in clinical practice, treatment with the three-drug combination as an FDC tablet resulted in a very high proportion of patients with a BP response and control, accompanied by a very low rate of ADRs.

Keywords: hypertension, clinical practice, fixed-dose combination, blood pressure, adverse drug reactions

\section{Introduction}

Hypertension is an independent cardiovascular risk factor, and each reduction of either $20 \mathrm{mmHg}$ in systolic (S) blood pressure (BP) or $10 \mathrm{mmHg}$ in diastolic (D) BP correlates with a twofold decrease in the likelihood of a fatal coronary event. ${ }^{1}$ To achieve the currently recommended BP goal of $<140 / 90 \mathrm{mmHg}$ (lower for specific patient populations), it is estimated that at least $25 \%$ of patients will require triple-agent therapy. ${ }^{2-4}$ The combined use of three different classes of antihypertensive drugs enables targeting of distinct biological pathways, thus enhancing efficacy compared with mono- or dual therapy. In addition, side effects caused by one drug class may be negated by the actions of another drug class that elicits opposing physiological compensatory mechanisms, resulting in a more favorable tolerability profile..$^{5-7}$ 
Among the five main classes of antihypertensive drugs, the combination of either an angiotensin converting enzyme (ACE) inhibitor or an angiotensin receptor blocker (ARB), which both inhibit the renin-angiotensin system, a calcium channel blocker, and a diuretic is the most commonly used triple-drug regimen. ${ }^{5,8-10}$ Compared with separate tablets for each drug, fixed-dose combination (FDC) tablets are associated with a higher rate of adherence to treatment, and, as a consequence, a greater proportion of patients attaining their BP goal over time. ${ }^{6,11,12}$ At the time of publication, only three fixed-dose triple-antihypertensive drug combination tablets were available, all containing the dihydropyridine calcium channel blocker amlodipine besylate and the thiazide diuretic hydrochlorothiazide (HCT). These two drugs are combined with the renin inhibitor aliskiren hemifumarate, the ARB valsartan, or the more recently developed ARB olmesartan medoxomil. ${ }^{13}$

A randomized 12-week clinical trial (TRINITY) of separate-tablet triple-drug therapy comprising olmesartan, amlodipine, and HCT demonstrated that the combination was well tolerated and efficacious in patients with moderate-to-severe hypertension, including those with cardiovascular disease, chronic kidney disease, and diabetes mellitus. ${ }^{14,15}$ In a 4-week, single-center, open-label study that evaluated the olmesartan/amlodipine/HCT FDC tablet in patients with inadequate BP control on single-, dual- or triple-agent therapy, all patients attained SBP goals with no reports of hypotension. ${ }^{16}$ Thus, clinical trials indicate that the three-drug combination of olmesartan, amlodipine, and HCT displays efficacy, tolerability, and safety when administered as separate tablets for each drug or, alternatively, as an FDC tablet.

The objective of the present noninterventional study was to determine whether findings from clinical trials can be applied to an unselected patient population in real-life clinical practice. This report covers the safety and effectiveness of the FDC tablet following 24 weeks of treatment, with an emphasis on the safety of the FDC according to age, cardiovascular risk profile, including diabetes mellitus, and concomitant medications.

\section{Methods}

\section{Study design}

Between November 2012 and December 2013, this binational, multicenter, noninterventional, open-label, prospective, noncontrolled observational study recruited 5,831 patients from primary care centers in Austria and Germany. The protocol was approved by the relevant ethics committees in Austria and Germany, and the study was performed according to the ethical standards of the Declaration of Helsinki. Signed informed consent was obtained from all patients prior to enrollment. It was registered with the "Verband Forschender Arzneimittelhersteller" (VFA).

\section{Patient population and schedule}

Adult ( $\geq 18$ years old) patients with essential hypertension (ie, with no known cause) were eligible for inclusion, providing that the olmesartan/amlodipine/HCT FDC tablet was indicated according to the summary of product characteristics, and treatment with the FDC had been initiated less than 2 weeks before the baseline visit. Exclusion criteria included contraindications to the FDC (eg, known hypersensitivity to any of the active substances of the FDC, to dihydropyridine derivatives, to other sulphonamide-derived substances or to any excipients of the compound), impaired renal function, treatment-resistant hypokalemia, hypercalcemia, hyponatremia, or symptomatic hyperuricemia, severely impaired liver function, cholelithiasis or biliary tract obstruction, as well as severe hypotension, (cardiogenic) shock, left ventricular obstruction, hemodynamically unstable heart failure after acute myocardial infarction, planned or existing treatment with the direct renin inhibitor aliskiren, and planned or current pregnancy.

The following five dose levels of the olmesartan/ amlodipine/HCT FDC tablet were used: 20/5/12.5 mg, $40 / 5 / 12.5 \mathrm{mg}, 40 / 5 / 25 \mathrm{mg}, 40 / 10 / 12.5 \mathrm{mg}$, and $40 / 10 / 25 \mathrm{mg}$. Up- or down-titration of the FDC tablet dose level was permitted at the discretion of the treating physician. The study had a planned follow-up period of $24 \pm 2$ weeks, with optional interim visits at $8 \pm 2$ weeks and $16 \pm 2$ weeks. At baseline, details of patient demographics and other characteristics were obtained, including time since diagnosis, relevant prior and concomitant diseases/risk factors, and concomitant antihypertensive and non-antihypertensive pharmacotherapies.

\section{Objectives}

The primary objective was to gain further insight into the safety profile of the olmesartan/amlodipine/HCT FDC tablet in clinical practice by documenting adverse drug reactions (ADRs) and their possible associations with concomitant pharmacotherapy. Secondary objectives were to evaluate the BP-lowering effectiveness of the FDC in clinical practice.

\section{ADRs}

All ADRs that occurred during the observation period and had a suspected causal relationship to the FDC tablet were recorded. Each ADR was coded using the Medical Dictionary 
for Regulatory Activities (latest version available at database lock). Subsequently, specific ADRs were translated into more general terms categorized by organ system. A serious ADR was recorded if any of the following events occurred: death, a life-threatening reaction, inpatient hospitalization or prolongation of existing hospitalization, persistent or significant disability, a medically significant reaction, or a congenital disease/birth defect.

\section{BP measurement}

Office sitting BP was measured at each visit using a calibrated standard sphygmomanometer and a cuff size appropriate for each patient. We recommended that measurements be performed after the patient had been seated for at least 5 minutes with the arm rested at the level of the heart. Physicians were advised to submit the mean BP value obtained from three separate readings.

\section{Statistical analyses}

Data were documented using a paper case report form and were entered into an electronic data capture system/project database. Exploratory descriptive statistical analysis was performed using Statistical Analysis System (SAS Institute, Cary, NC, USA). The safety set included all patients who received at least one dose of the FDC. The full analysis set, which was used to analyze efficacy, comprised all patients in the safety set who also had valid and available data on SBP and DBP at baseline as well as at least one postbaseline visit. Continous parameters are described by means of absolute numbers \pm standard deviation (SD; including the number of nonmissing and missing observations), while for discrete parameters, frequencies (\%) are presented.

\section{Results}

A total of 5,831 patients were enrolled, of whom 451 and 5,380 were cared for by physicians in Austria and Germany, respectively. The safety set comprised 5,831 patients, and the full analysis set included 5,818 patients.

\section{Patient characteristics}

The mean age of patients was $63.5( \pm 11.79)$ years, including $50.5 \%$ aged between 40 and 65 years, $29.2 \%$ between 65 and 75 years, and $18.1 \%$ aged $\geq 75$ years. There were fewer females $(47.0 \%)$ than males $(53.0 \%)$ in the study population. The mean body mass index was $29.4( \pm 4.89) \mathrm{kg} / \mathrm{m}^{2}$, and $36.9 \%$ of patients were obese $\left(\geq 30 \mathrm{~kg} / \mathrm{m}^{2}\right)$. Essential hypertension was the indication for $97.9 \%$ of patients, and the time since diagnosis was more than 5 years for $47.5 \%$ of patients,
$1-5$ years for $30.1 \%$ of patients, less than 1 year for $11.1 \%$ of patients, and unknown for $6.5 \%$ of patients ( $4.8 \%$ missing). Risk factors were present in approximately $70 \%$ of the study population, with $54.7 \%$ having one or two risk factors, and $10.2 \%$ having three risk factors or more. The most common risk factors were diabetes mellitus (29.4\%), metabolic syndrome $(21.1 \%)$, smoking $(17.8 \%)$, left ventricular hypertrophy (9.9\%), and cardiac failure (7.4\%; Table 1). In $24.3 \%$ of the patients "other risk factors" were reported.

\section{Treatment patterns}

Prior to the study, ARBs, calcium channel blockers, and diuretics were being administered to $25.8 \%, 33.2 \%$, and $44.9 \%$ of the study population, while ACE inhibitors, betablockers, renin inhibitors, and other antihypertensive drug classes were being taken by $52.4 \%, 39.4 \%, 0.9 . \%$, and $6.8 \%$ of patients respectively. With regard to the number of antihypertensive drugs and the use of separate tablets or an FDC, the majority of patients were receiving antihypertensive monotherapy (25.1\%), separate dual-agent therapy (23.9\%), or separate triple-agent therapy $(20.3 \%)$. The most common reason for switching to the FDC was a perceived lack of efficacy (87.7\%; Table 2).

Table I Patient demographics at baseline ( $n=5,83$ I, safety set)

\begin{tabular}{|c|c|}
\hline Parameters & Mean \pm SD or $\%$ \\
\hline Age in years & $63.5 \pm 11.79$ \\
\hline$\geq 40$ to $<65$ years & 50.5 \\
\hline$\geq 65$ years & 29.2 \\
\hline$\geq 75$ years & I8. I \\
\hline Female sex & 47.0 \\
\hline Body mass index $\left(\mathrm{kg} / \mathrm{m}^{2}\right)$ & $29.4 \pm 4.89$ \\
\hline Essential hypertension & 97.9 \\
\hline \multicolumn{2}{|l|}{ Time since diagnosis } \\
\hline Unknown & 6.5 \\
\hline$<$ I year & II.I \\
\hline I to 5 years & 30.1 \\
\hline$>5$ years & 47.5 \\
\hline \multicolumn{2}{|l|}{ Risk factors } \\
\hline Diabetes mellitus & 29.4 \\
\hline Metabolic syndrome & 21.1 \\
\hline Smoking & 17.8 \\
\hline Left ventricular hypertrophy & 9.9 \\
\hline Cardiac failure & 7.4 \\
\hline Renal dysfunction & 4.3 \\
\hline Stroke/TIA & 4.1 \\
\hline Stable angina pectoris & 4.0 \\
\hline Myocardial infarction & 3.6 \\
\hline Peripheral artery disease & 3.5 \\
\hline Hepatic impairment & 1.3 \\
\hline Other unspecified risk factors & 24.3 \\
\hline
\end{tabular}

Abbreviations: SD, standard deviation; TIA, transient ischemic attack. 
Table 2 Types and number of antihypertensive premedications, and the reason for switching to the FDC tablet ( $n=5,83$ I, safety set)

\begin{tabular}{ll}
\hline Prior antihypertensive medication & Frequency (\%) \\
\hline By drug class* & \\
ACE inhibitor & 52.4 \\
ARB & 25.8 \\
Beta-blocker & 39.4 \\
Calcium channel blocker & 33.2 \\
Diuretic & 44.9 \\
Renin inhibitor & 0.9 \\
Other antihypertensive drug classes & 6.8 \\
By number/separate tablets or FDC & \\
None & 6.6 \\
Monotherapy & 25.1 \\
Separate dual-agent therapy & 23.9 \\
FDC dual-agent therapy & 14.0 \\
Separate triple-agent therapy & 20.3 \\
FDC triple-agent therapy & 0.7 \\
More than three agents & 9.5 \\
Reason for switching to the FDC* & \\
Lack of efficacy & 87.7 \\
Noncompliance & 20.3 \\
Simplification of therapy & 45.1 \\
Wish of the patient & 11.8 \\
Other & 6.5 \\
\hline
\end{tabular}

Note: *Multiple choices were possible.

Abbreviations: $A C E$, angiotensin converting enzyme; ARB, angiotensin receptor blocker; FDC, fixed-dose combination.

During the follow-up period ( $24 \pm 2$ weeks), mean exposure to the FDC tablet was $180.7( \pm 41.16)$ days. The mean daily dose was 32.8 ( \pm 9.48$) \mathrm{mg}$ for olmesartan, $6.2( \pm 2.16) \mathrm{mg}$ for amlodipine, and $15.0( \pm 5.07) \mathrm{mg}$ for HCT. After baseline and at the final assessment, the most frequently prescribed dose regimen was $20 / 5 / 12.5 \mathrm{mg}$, which was administered to $37.4 \%$ and $32.0 \%$ of patients at the two respective time points, followed by $40 / 5 / 12.5 \mathrm{mg}(29.3 \%$ and $29.1 \%$ of patients, respectively), $40 / 10 / 25 \mathrm{mg}$ (13.4\% and $15.9 \%$ of patients, respectively), $40 / 10 / 12.5 \mathrm{mg}(10.4 \%$ and $13.0 \%$ of patients, respectively), and 40/5/25 $\mathrm{mg}(9.6 \%$ and $9.9 \%$ of patients, respectively; Table 3 ).

Concomitant antihypertensive treatments were being administered to $39.2 \%$ and $35.2 \%$ of patients at baseline and at follow-up (V4), respectively, with beta-blockers being prescribed to $30.5 \%$ and $29.2 \%$ of patients at the two respective time points, and all other classes of antihypertensive agent being prescribed to $<10 \%$ of the study population. Concomitant non-antihypertensive medications were being taken by $66.1 \%$ and $66.1 \%$ of patients at baseline and follow-up, respectively. These agents included lipidlowering drugs $(32.3 \%$ and $32.6 \%$, at the two respective time points), oral antidiabetic agents $(22.0 \%$ and $22.7 \%$, respectively), and acetylsalicylic acid(19.1\% and 19.5\%, respectively),
Table 3 Treatment regimens at baseline and follow-up $(n=5,83$ I, safety set)

\begin{tabular}{|c|c|c|}
\hline & $\begin{array}{l}\text { Baseline, } \\
\text { N (\%) }\end{array}$ & $\begin{array}{l}\text { 24-week follow-up, } \\
\text { N (\%) }\end{array}$ \\
\hline \multicolumn{3}{|l|}{ Dose level of FDC tablet $(\mathrm{mg})$} \\
\hline $20 / 5 / 12.5$ & $2,179(37.4)$ & I,863 (31.9) \\
\hline $40 / 10 / 12.5$ & 779 (13.4) & 925 (I5.9) \\
\hline $40 / 10 / 25$ & $604(10.4)$ & $756(13.0)$ \\
\hline $40 / 5 / 12.5$ & I,7II (29.3) & I,699 (29.1) \\
\hline $40 / 5 / 25$ & $558(9.6)$ & $576(9.9)$ \\
\hline Unknown & $0(0.0)$ & $12(0.2)$ \\
\hline Antihypertensive & $2,288(39.2)$ & I,980 (35.2) \\
\hline \multicolumn{3}{|l|}{ concomitant medications } \\
\hline ACE inhibitor & $319(5.5)$ & $91(1.6)$ \\
\hline $\mathrm{ARB}$ & $148(2.5)$ & $44(0.8)$ \\
\hline Beta-blocker & I,778 (30.5) & $\mathrm{I}, 64 \mathrm{I}(29.2)$ \\
\hline Calcium channel blocker & $263(4.5)$ & $|2|(2.2)$ \\
\hline Diuretic & $457(7.8)$ & $286(5.1)$ \\
\hline $\begin{array}{l}\text { Other antihypertensive } \\
\text { drug classes }\end{array}$ & $297(5.1)$ & $293(5.2)$ \\
\hline \multicolumn{3}{|l|}{ concomitant medications } \\
\hline Lipid-lowering & I,885 (32.3) & I,834 (32.6) \\
\hline Oral antidiabetics & $1,282(22.0)$ & I,273 (22.7) \\
\hline Aspirin & $\mathrm{I}, \mathrm{I} 12(19.1)$ & $\mathrm{I}, 098(19.5)$ \\
\hline Psychotropic agents & $420(7.2)$ & $419(7.5)$ \\
\hline NSAID/COX II antagonists & $373(6.4)$ & $373(6.6)$ \\
\hline Insulin & $360(6.2)$ & $35 I(6.2)$ \\
\hline Anticoagulants & $26 \mid(4.5)$ & $26 \mid(4.6)$ \\
\hline Others & I,423 (24.4) & I,332 (23.7) \\
\hline
\end{tabular}

Note: Values are expressed as the number of patients $(\mathrm{N})$ and the frequency $(\%)$. Abbreviations: ACE, angiotensin converting enzyme; ARB, angiotensin receptor blocker; COX, cyclo-oxygenase; FDC, fixed-dose combination; NSAID, nonsteroidal anti-inflammatory drug.

as well as psychotropic agents (7.2\% and 7.5\%, respectively), nonsteroidal anti-inflammatory drugs (NSAIDs)/ cyclo-oxygenase (COX) II antagonists (6.4\% and 6.6\%, respectively), insulin ( $6.2 \%$ and $6.2 \%$, respectively), anticoagulants ( $4.5 \%$ and $4.6 \%$, respectively), and other types of non-antihypertensive treatment $(24.4 \%$ and $23.7 \%$, respectively; Table 3).

\section{Adverse drug reactions, concomitant treatment, and risk factors}

Among the 5,831 patients in the safety data set, 1.23\% $(n=75)$ had at least one ADR, including $0.09 \%(n=5)$ with at least one serious ADR, and $0.82 \%(n=48)$ with at least one ADR that led to study discontinuation (Table 4). The most frequent types of ADR by primary organ system were general disorders and administration site conditions ( $0.6 \%$; predominantly peripheral edema, $0.3 \%$ ), skin and subcutaneous tissue disorders $(0.19 \%)$, nervous system disorders $(0.17 \%$; primarily dizziness, $0.12 \%)$, vascular disorders $(0.15 \%$; the 
Table 4 Frequency of adverse drug reactions ( $n=5,83$ I, safety set) overall and according to primary organ system, including all individual ADRs reported by more than two patients

\begin{tabular}{lll}
\hline Type of ADR & & $\begin{array}{l}\text { Number of } \\
\text { patients (\%) }\end{array}$ \\
\hline $\begin{array}{l}\text { At least one ADR } \\
\text { At least one serious ADR }\end{array}$ & $75(\mathrm{I.23})$ \\
$\begin{array}{l}\text { At least one ADR that led to } \\
\text { study discontinuation }\end{array}$ & $5(0.09)$ \\
$\begin{array}{l}\text { ADRs by primary } \\
\text { organ system }\end{array}$ & Number of & Number of \\
& ADRs & patients with at \\
least one ADR (\%)
\end{tabular}

Abbreviation: ADR, adverse drug reaction.
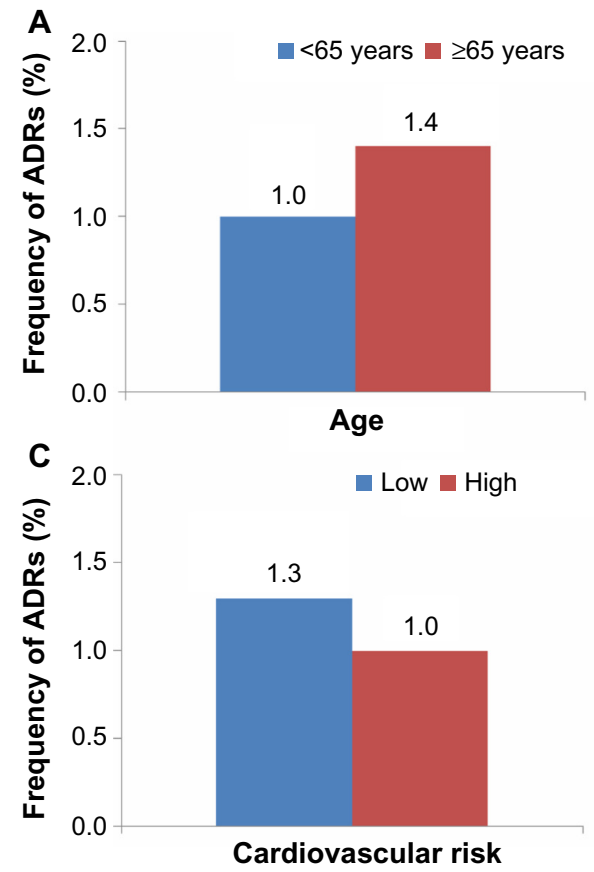

majority of which were hypotension, $0.14 \%$ ), and gastrointestinal disorders $(0.10 \%)$. Further analyses to determine the influence of age ( $<65$ years versus $\geq 65$ years; Figure $1 \mathrm{~A}$ ), diabetes mellitus (no/yes; Figure 1B), and cardiovascular risk (low/high; Figure 1C) demonstrated that the rate of ADRs was lower among patients aged $<65$ years $(1.0 \%$ versus $1.4 \%)$, those with diabetes mellitus $(0.7 \%$ versus $1.5 \%)$, and those with low cardiovascular risk $(1.3 \%$ versus $1.0 \%)$. For patients who received any type of concomitant medication (antihypertensive and/or non-antihypertensive), the rate of ADRs was higher (1.4\%) than for those who received only the FDC tablet $(0.7 \%$; Figure $1 \mathrm{D})$.

\section{BP-lowering effectiveness}

Using data from the 5,818 patients in the full analysis set, the mean SBP and DBP changed from $162.1( \pm 17.01) \mathrm{mmHg}$ and $93.6( \pm 10.56) \mathrm{mmHg}$, respectively, at baseline, to 133.1 $( \pm 10.38) \mathrm{mmHg}$ and $79.7( \pm 7.02) \mathrm{mmHg}$, respectively, at the 24 weeks follow-up, corresponding to a mean reduction in SBP/DBP of 28.8/13.9 mmHg (Figure 2). The frequency of patients with $\mathrm{BP}<140 / 90 \mathrm{mmHg}$ was $5.1 \%$ at baseline, rising to $67.5 \%$ at follow-up. A BP response, defined as SBP $<140 \mathrm{mmHg}$ and DBP $<90 \mathrm{mmHg}$ or a change from baseline of $\geq 20 \mathrm{mmHg}$ in $\mathrm{SBP}$ and/or $\geq 10 \mathrm{mmHg}$ in DBP, was observed in $94.2 \%$ of patients at follow-up. With regard to the severity of hypertension, $16.1 \%, 39.0 \%, 19.0 \%$,

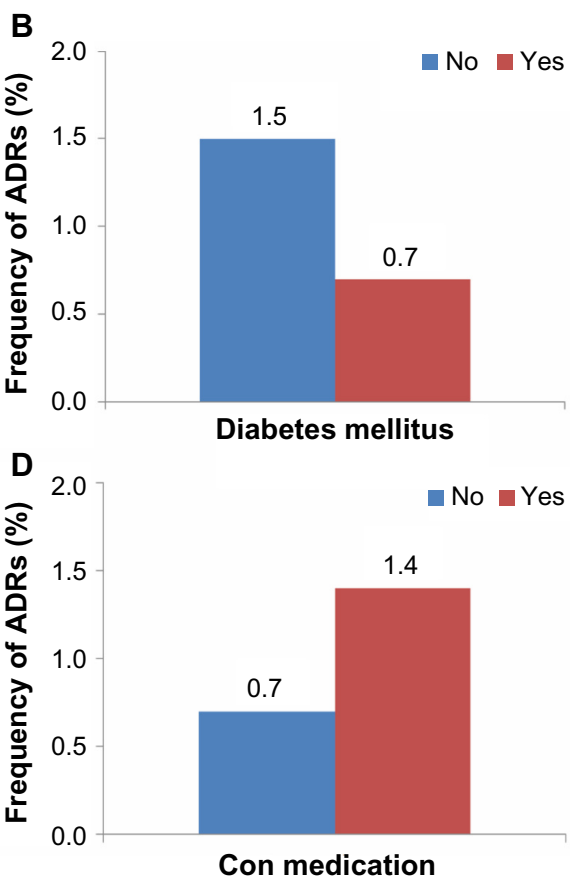

Figure I Frequency of adverse drug reactions ( $n=5,83$ I, safety set).

Notes: (A) Age, (B) diabetes mellitus, (C) cardiovascular risk, and (D) concomitant medications.

Abbreviations: ADRs, adverse drug reactions; Con, concomitant. 


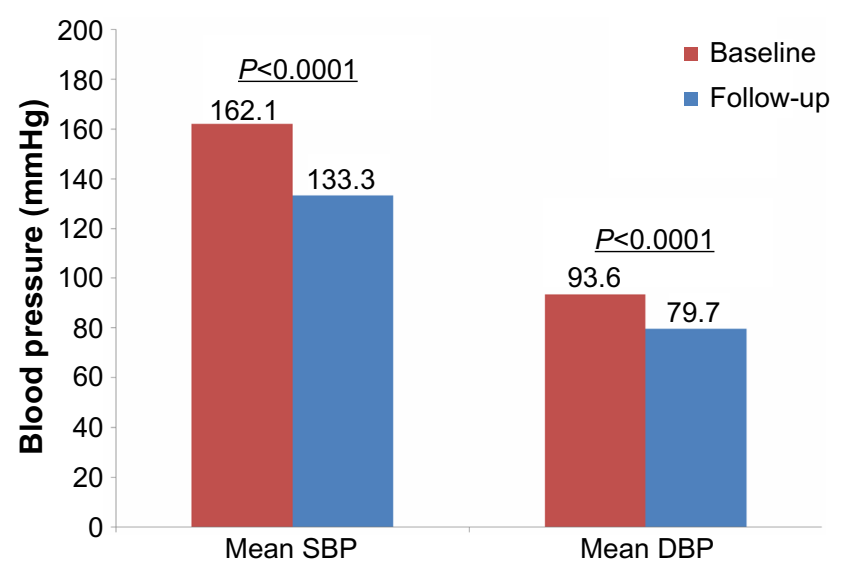

Figure 2 Mean change in systolic and diastolic blood pressure from baseline to the 24-week follow-up ( $n=5,8 \mid 8$, full analysis set).

Note: Mean differences between SBP and DBP are significant with a $P$-value of $<0.000$ I.

Abbreviations: SBP, systolic blood pressure; DBP, diastolic blood pressure.

and $20.8 \%$ of patients were considered to have grade 1 , grade 2, grade 3, and isolated systolic hypertension at baseline, respectively, compared with $9.1 \%, 1.3 \%, 0.4 \%$, and $22.6 \%$ of patients with each respective class of hypertension at the follow-up visit (Figure 3).

\section{Discussion}

The results of the present study are in accordance with those from randomized controlled clinical trials that demonstrate that the FDC olmesartan/amlodipine/HCT tablet is associated

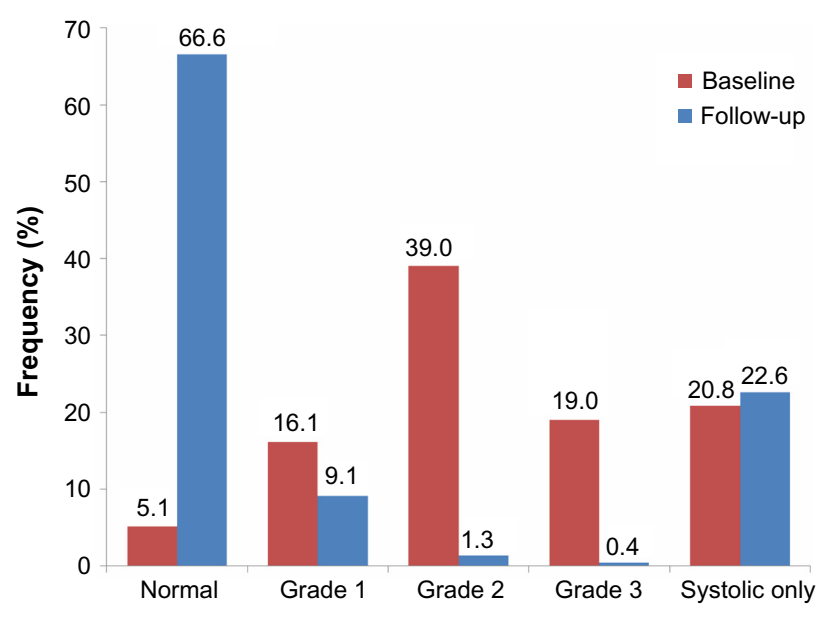

Figure 3 Change in the severity of hypertension from baseline to the 24-week follow-up according to the $2007 \mathrm{ESC} / \mathrm{ESH}$ classification ( $n=5,8 \mid 8$, full analysis set). Notes: "Normal" includes all patients with optimal systolic/diastolic BP $(<\mid 20 / 80 \mathrm{mmHg})$, normal BP (I20-129 and/or 80-85 mmHg), and normal-high BP (I30-139 and/or 85-89 mmHg). Grades I, 2, and 3 hypertension were defined as systolic and/or diastolic BPs of $140-159$ and/or $90-99 \mathrm{mmHg}$, respectively, 160-179 and/or 100-109 mmHg, respectively, and $\geq 180$ and/or $\geq 110 \mathrm{mmHg}$, respectively. "Systolic only" corresponds to isolated systolic hypertension ( $\geq 140 \mathrm{mmHg}$ for systolic BP and $<90 \mathrm{mmHg}$ for diastolic BP).

Abbreviations: ESC, European Society of Cardiologists; ESH, European Society of Hypertension; BP, blood pressure. with very few ADRs, even among patients who are elderly, have diabetes mellitus or other cardiovascular risk factors, and those receiving concomitant medications. Furthermore, after $24 \pm 2$ weeks of treatment with the FDC tablet, $68 \%$ of patients uncontrolled at baseline had $\mathrm{BP}<140 / 90 \mathrm{mmHg}$, and $94 \%$ of patients had a favorable BP response. Thus, this study indicates that data from clinical trials provide an accurate translation of the safety and efficacy of the FDC olmesartan/ amlodipine/HCT tablet in a real-life environment.

\section{Patient demographics}

One of the key advantages of an observational study is the inclusion of patients who would be excluded from clinical trials, resulting in a broader patient population that is more representative of that in real-life clinical practice. This is illustrated by considering the differences in baseline demographics between patients included in the present study and those who received the three-drug olmesartan, amlodipine, HCT combination in the TRINITY clinical trial. ${ }^{14}$ Compared with the three-drug arm of the TRINITY study, patients in the present study were on average older (63.5 years versus 54.7 years), and there was a much higher proportion of patients aged over 65 years $(47.3 \%$ versus $22.5 \%)$. Obesity was less prevalent $(36.9 \%$ versus $61.7 \%)$, but the frequency of diabetes mellitus was almost twofold higher in the present study $(29.4 \%$ versus $15.3 \%) .{ }^{14}$

\section{ADRs and BP-lowering efficacy}

The rate of ADRs in the present study was low, with the most frequent ADRs being peripheral edema, dizziness, and hypotension, which is consistent with the safety profile of the three-drug combination in clinical trials..$^{14,16,17}$ In the TRINITY clinical trial, in which all patients received 12 weeks' treatment with the highest dose level of each drug (40/10/25 mg), dizziness and peripheral edema were the most common ADRs, followed by headache and fatigue. ${ }^{14}$ Similarly, in the 4-week study of the FDC tablet, the most prevalent treatment-emergent adverse events were peripheral edema and dizziness. ${ }^{16}$ With regard to effectiveness, attainment of a BP goal of $<140 / 90 \mathrm{mmHg}$ was experienced by $67 \%$ of patients in the present study, $70 \%$ in the TRINITY clinical trial, ${ }^{14}$ and $78 \%$ in a 36 -week open-label extension study that assessed the three-drug separate-tablet combination of olmesartan, amlodipine, and HCT. ${ }^{17}$ Of note, subanalyses of the TRINITY trial revealed that the rate of BP goal attainment was lower among patients with diabetes mellitus (41.1\%) or chronic cardiovascular disease (38.9\%). ${ }^{15}$ Given that the proportion of patients with diabetes mellitus was approximately 
twofold higher in the present study, this may have contributed to the slightly lower rate of BP goal attainment.

\section{ADRs and patient subpopulations}

Results from this study indicate that the rate of ADRs was only slightly increased among patients who were over 65 years of age, and those with high cardiovascular risk. A study by Weir et $\mathrm{al}^{18}$ of an FDC tablet of olmesartan/ amlodipine with separate-pill HCT also indicated that age did not have a relevant influence on the ADR rate. Drugrelated hypotension and orthostatic hypotension, however, occurred less frequently in patients aged greater than 65 years $(2.2 \%$ and $0.0 \%)$ compared with under 65 years $(2.3 \%$ and $0.3 \%)$. Although in the present study, subanalyses according to the types of ADRs that were observed in elderly, high-risk, or diabetic patients were not conducted, data from other clinical trials provide some insight into the ADRs that occur more frequently in these patient populations. In the TRINITY clinical trial, subanalyses demonstrated that the most frequent ADRs among patients with diabetes mellitus or chronic cardiovascular disease were peripheral edema and dizziness, which corresponds to findings for the total patient population in both studies. ${ }^{15}$

Perhaps more surprisingly than the findings in elderly and high-risk patients, the rate of ADRs in the present study was $0.7 \%$ lower in patients with diabetes than those without. In the absence of any obvious explanation, it may be hypothesized that patients with diabetes mellitus had greater adherence to treatment because of the need for routine antidiabetic medication. Another possibility is that the combination of oral antidiabetic drugs with antihypertensive medications may have a complementary effect on the tolerability profile of the two types of treatments. Related to this theory, a study by Ogihara et $\mathrm{al}^{19}$ in patients with hypertension and diabetes mellitus demonstrated that the reduction in insulin resistance induced by the thiazolidinedione troglitazone was associated with enhanced BP control.

\section{Concomitant medications}

As in other clinical trials of antihypertensive agents, patients in the TRINITY clinical trial were not allowed to receive concomitant medications that may alter BP. In contrast, in the present study, $35 \%-39 \%$ of patients were receiving antihypertensive concomitant medications, and about $66 \%$ were receiving concomitant non-antihypertensive medications. These data are relevant because of the potential impact of concomitant medications on the safety, tolerability, and efficacy of the FDC. The rate of ADRs in the present study was only $0.5 \%$ higher in patients prescribed any type of concomitant medication compared with those who only received the FDC tablet. In addition, no previously unidentified drug interactions were observed. This suggests that the FDC may be safely administered in combination with a variety of other drugs. Nevertheless, further analyses are warranted to determine whether any individual concomitant medication had a noticeable effect on ADRs. For example, it would be of interest to know if the $0.5 \%$ increase in ADRs corresponds to a specific type of ADR or a specific type of concomitant medication.

\section{Strengths and limitations}

Because this was an observational study, it allows a more realistic evaluation of the effectiveness of the FDC tablet when applied in a real-life setting. ${ }^{20,21}$ In addition, this study included elderly, high-risk, and diabetic patients, as well as those receiving concomitant medications. Thus, this report represents an important source of information to inform clinical practice and should help to better define optimal treatment strategies for patient populations that are prevalent in clinical practice, but underrepresented in clinical trials. Limitations of this study include the lack of adjustment for confounding variables and biases in the patient population. For example, one confounding variable is the use of additional medications, both antihypertensive and non-antihypertensive. Also, data from this study are specific to the central European demographic, but it is presently unknown whether these results hold true for different ethnic backgrounds, health systems, and providers. Finally, it is known that the reporting of adverse events is less complete than in clinical trials, potentially leading to an underestimation of the true hazard.

\section{Conclusion}

This study reveals that in real-life clinical practice, the FDC olmesartan/amlodipine/HCT tablet was associated with very few ADRs combined with two-thirds of patients attaining a BP goal of $<140 / 90 \mathrm{mmHg}$. These data should help to inform clinical practice, and indicate that the higher level of treatment adherence associated with an FDC tablet may translate into superior patient outcomes compared with multiple separate tablets. Furthermore, this study demonstrates that treatment with three different classes of antihypertensive drug, with or without the use of concomitant drugs and using the most appropriate dose regimen for each patient, resulted in $94 \%$ of patients attaining a BP response, and a clinically relevant reduction in the risk of cardiovascular events might be expected. 


\section{Author contributions}

Peter Bramlage, Eva-Maria Fronk, Wolf-Peter Wolf, Rüdiger Smolnik, and Roland E Schmieder designed the study. Eva-Maria Fronk was responsible for the statistical analyses. Peter Bramlage and Gemma Sutton designed the analyses, interpreted the findings and drafted the first version of the manuscript. All authors revised the article for important intellectual content. All authors approved the final version of the manuscript that was submitted. The authors are accountable for all aspects of the work in ensuring that questions related to the accuracy or integrity of any part of the work are appropriately investigated and resolved.

\section{Disclosure}

The study was funded and conducted by Daiichi Sankyo Europe GmbH, Munich, Germany. Peter Bramlage and Roland E Schmieder have received research funds and consultancy honoraria from Daiichi Sankyo. Eva-Maria Fronk, Wolf-Peter Wolf, and Rüdiger Smolnik are employees of Daiichi Sankyo. Gemma Sutton has no conflict of interest related to this manuscript. The authors report no other conflicts of interest in this work.

\section{References}

1. Collins R, MacMahon S. Blood pressure, antihypertensive drug treatment and the risks of stroke and of coronary heart disease. Br Med Bull. 1994;50:272-298.

2. Mancia G, Laurent S, Agabiti-Rosei E, et al. Reappraisal of European guidelines on hypertension management: a European Society of Hypertension Task Force document. J Hypertens. 2009;27:2121-2158.

3. Gradman AH. Rationale for triple-combination therapy for management of high blood pressure. J Clin Hypertens (Greenwich). 2010;12: 869-878.

4. Wright JT Jr, Dunn JK, Cutler JA, et al. Outcomes in hypertensive black and nonblack patients treated with chlorthalidone, amlodipine, and lisinopril. JAMA. 2005;293:1595-1608.

5. Salahuddin A, Mushtaq M, Materson BJ. Combination therapy for hypertension 2013: an update. J Am Soc Hypertens. 2013;7:401-407.

6. Kizilirmak P, Berktas M, Uresin Y, Yildiz OB. The efficacy and safety of triple vs dual combination of angiotensin II receptor blocker and calcium channel blocker and diuretic: a systematic review and meta-analysis. J Clin Hypertens (Greenwich). 2013;15:193-200.

7. Neutel JM, Smith DH. Hypertension management: rationale for triple therapy based on mechanisms of action. Cardiovasc Ther. 2013;31: $251-258$.
8. Mancia G, Fagard R, Narkiewicz K, et al. 2013 ESH/ESC guidelines for the management of arterial hypertension: the Task Force for the Management of Arterial Hypertension of the European Society of Hypertension (ESH) and of the European Society of Cardiology (ESC). Eur Heart J. 2013;34:2159-2219.

9. Kjeldsen SE, Aksnes TA, Ruilope LM. Clinical implications of the 2013 ESH/ESC hypertension guidelines: targets, choice of therapy, and blood pressure monitoring. Drugs R D. 2014;14:31-43.

10. de la Sierra A, Barrios V. Blood pressure control with angiotensin receptor blocker-based three-drug combinations: key trials. Adv Ther. 2012;29:401-415.

11. Panjabi S, Lacey M, Bancroft T, Cao F. Treatment adherence, clinical outcomes, and economics of triple-drug therapy in hypertensive patients. J Am Soc Hypertens. 2013;7:46-60.

12. Bramlage P, Hasford J. Blood pressure reduction, persistence and costs in the evaluation of antihypertensive drug treatment-a review. Cardiovas Diabetol. 2009;8:18.

13. Drugs@FDA [homepage on the Internet] Silver Spring: U.S. Food and Drug Administration; 2014. Available from: http://www.accessdata.fda. gov/scripts/cder/drugsatfda/. Accessed November 21, 2014.

14. Oparil S, Melino M, Lee J, Fernandez V, Heyrman R. Triple therapy with olmesartan medoxomil, amlodipine besylate, and hydrochlorothiazide in adult patients with hypertension: the TRINITY multicenter, randomized, double-blind, 12-week, parallel-group study. Clin Ther. 2010;32:1252-1269.

15. Kereiakes DJ, Chrysant SG, Izzo JL Jr, et al. Olmesartan/amlodipine/ hydrochlorothiazide in participants with hypertension and diabetes, chronic kidney disease, or chronic cardiovascular disease: a subanalysis of the multicenter, randomized, double-blind, parallel-group TRINITY study. Cardiovasc Diabetol. 2012;11:134.

16. Punzi HA. Efficacy and safety of olmesartan/amlodipine/ hydrochlorothiazide in patients with hypertension not at goal with mono, dual or triple drug therapy: results of the CHAMPiOn study. Ther Adv Cardiovasc Dis. 2014;8:12-21.

17. Volpe M, de la Sierra A, Ammentorp B, Laeis P. Open-label study assessing the long-term efficacy and safety of triple olmesartan/ amlodipine/hydrochlorothiazide combination therapy for hypertension. Adv Ther. 2014;31:561-574.

18. Weir MR, Shojaee A, Maa JF. Efficacy of amlodipine/olmesartan medoxomil \pm hydrochlorothiazide in patients aged $\geq 65$ or $<65$ years with uncontrolled hypertension on prior monotherapy. Postgrad Med. 2013;125:124-134.

19. Ogihara T, Rakugi H, Ikegami H, Mikami H, Masuo K. Enhancement of insulin sensitivity by troglitazone lowers blood pressure in diabetic hypertensives. Am J Hypertens. 1995;8:316-320.

20. Wang OJ, Krumholz HM. Clinical trial participation: are we studying the patients we are trying to treat? Eur J Heart Failure. 2009;11: 1021-1022.

21. Nallamothu BK, Hayward RA, Bates ER. Beyond the randomized clinical trial: the role of effectiveness studies in evaluating cardiovascular therapies. Circulation. 2008;118:1294-1303.
Vascular Health and Risk Management

\section{Publish your work in this journal}

Vascular Health and Risk Management is an international, peerreviewed journal of therapeutics and risk management, focusing on concise rapid reporting of clinical studies on the processes involved in the maintenance of vascular health; the monitoring, prevention and treatment of vascular disease and its sequelae; and the involvement of

\section{Dovepress}

metabolic disorders, particularly diabetes. This journal is indexed on PubMed Central and MedLine. The manuscript management system is completely online and includes a very quick and fair peer-review system, which is all easy to use. Visit http://www.dovepress.com/ testimonials.php to read real quotes from published authors. 